Research Article

\title{
The Prognostic Value of Traditional Chinese Medicine Symptoms in Acute Ischemic Stroke: A Pilot Study
}

\author{
Jia Xu, Jian Pei $\mathbb{D}^{D}$, Qin-hui Fu, and Yi-jun Zhan \\ Department of Acupuncture, LongHua Hospital, Shanghai University of Traditional Chinese Medicine, Shanghai, China \\ Correspondence should be addressed to Jian Pei; longhuaacup@aliyun.com
}

Received 20 December 2019; Revised 14 February 2020; Accepted 24 June 2020; Published 15 July 2020

Academic Editor: Arthur De Sá Ferreira

Copyright (c) 2020 Jia Xu et al. This is an open access article distributed under the Creative Commons Attribution License, which permits unrestricted use, distribution, and reproduction in any medium, provided the original work is properly cited.

\begin{abstract}
Background. Stroke scales of traditional Chinese medicine (SSTCM) are promoted for use in the early prognosis. The current lines of evidence to support their performance evaluation are uneven. This pilot study aimed to investigate the correlation between traditional Chinese medicine (TCM) symptoms in the early stages of acute ischemic stroke and the prognosis of motor dysfunction through one-year of follow-up. Methods. Three hundred and fifteen patients were retrospected at Longhua Hospital from January 2016 to December 2017. All patients had received standard treatments combined with acupuncture therapy, including both electroacupuncture and scalp acupuncture for a median course of five months. The observed outcomes were the Fugl-Meyer assessment (FMA), the modified Barthel index (MBI), and the modified Rankin scale (mRS) at one-year follow-up after stroke onset by multiple linear regression analysis combined with ROC curves. Results. The favorable outcome rate was $74.3 \%$, with the recurrence rate of $20.3 \%$ in the follow-up. In multiple linear regression, $10 \mathrm{TCM}$ symptoms (MBI regression model) were related to the prognosis of MBI (DW 1.409, Ad. $R^{2} 0.654$ ) and 10 TCM symptoms (FMA regression model) were related to the FMA outcome (DW 1.446, Ad $R^{2}$ 0.620). The two models were selected to have nine repeated symptoms (repeated model). In the ROC curves, the three models were compared with the NIHSS score, and the MBI regression model reflected the highest efficiency. Conclusions. The combination of 10 TCM symptoms, once onset occurred, including hemiplegia, restlessness, hemianesthesia, short breath, headache, constipation, night sweat, tinnitus, thirsty, and gurgling with sputum, may affect the recovery of motor dysfunction. Furthermore, the improvements of TCM symptoms dynamically after treatment would be observed in a large prospective cohort. This trial is registered with NCT01806233.
\end{abstract}

\section{Background}

Stroke is associated with the highest disability-adjusted lifeyears (DALYs) cost, as the first fatal disease in China, with over two million new cases annually [1], accounting for $40 \%$ of the global total [2]. With the change of environment and unhealthy lifestyle, the incidence among young and middleaged adults (20-64 years old) displays an increasing trend [3]. The estimated onset age of stroke in China is about 10 years earlier than that in developed countries [1]. Typically, motor dysfunction is the most severe neurological deficit after stroke, with a disability rate of as high as $60 \%$ [4]. Given that China had the highest morbidity and mortality rates globally, more vigorous and effective actions are needed to reduce the burden of stroke.
Considering the high disability and recurrence rates of ischemic stroke, early prognosis plays an important role in making the treatment plans and evaluating clinical efficacy. With the progress of bio-psycho-social model of medicine, health-related quality of life (hr-QOL) and patient-reported outcomes (PROs) are regarded as the gold standards for evaluating the quality of life and subjective feelings of patients [5]. SSTCM, established under the guidance of TCM, and through the combined use of the four diagnostic methods, pay more attention to the unity of the body and spirit, pattern differentiation, and personal physique so as to adapt to Chinese conditions. However, the current evidence showed the performance evaluation of TCM scales is uneven [6]. Scales in general accepted standards have not been applied as early predictive indicators of stroke outcome in 
the prognostic scale [7]. Typically, some early prognostic symptoms are important for the development of precision medicine, quality of life, and prognosis of stroke, which need to be testified in the progress of medical practice.

With the rapid development of big data, real-world evidence is playing an increasing role in healthcare decisions. As a complementary role of clinical evidence, realworld data can come from various clinical practices directly, without deliberate selection of subjects and excessive manual intervention [8]. Consequently, real-world studies can reconstruct the real clinical scene maximumly in order to investigate clinical problems, especially in etiologic and prognoses research. This retrospective study, as one part of real-word studies, can provide evidence for evidence-based practice.

This pilot study aimed to investigate the correlation between TCM symptoms in the early stages of acute ischemic stroke and the prognosis of motor dysfunction. The clinical cases were retrospectively reviewed to assess the motor dysfunction in FMA through a one-year follow-up so as to provide evidence for the establishment of prognostic SSTCM.

\section{Materials and Methods}

2.1. Study Design. In this retrospective study, the inpatient electronic medical records database at Longhua Hospital Affiliated to Shanghai University of Traditional Chinese Medicine was accessed to analyze the secondary data.

2.2. Study Subjects. 1650 cases with acute ischemic stroke were recruited between January $1^{\text {st }}, 2016$, and December $31^{\text {st }}$, 2017, from five inpatient departments in Longhua Hospital: department of TCM, neurology department, emergency ward, special ward, and geriatrics department. All patients received the standard treatments in accordance with the Chinese guidelines for the diagnosis and treatment of acute ischemic stroke [9].

\subsection{Diagnostic Criteria}

(1) Ischemic stroke was diagnosed with reference to the criteria of cerebral arterial thrombosis from the Chinese Stroke Society [9]

(2) TCM diagnostic criteria of apoplexy referred to the "Standards of Syndrome-Differentiated Diagnosis of Apoplexy" in Chinese medicine [10]

\subsection{Inclusion Criteria}

(1) Adults patients should meet the diagnostic criteria of acute ischemic stroke and apoplexy mentioned previously

(2) Ischemic stroke can be confirmed by a brain computed tomography (CT) or magnetic resonance imaging (MRI) scan

(3) Patients who experienced an acute stroke within $24 \mathrm{~h}$ were included.
(4) Stroke patients with limb motor dysfunction were recorded

(5) The National Institutes of Health Stroke Scale (NIHSS) scores, the Fugl-Meyer Assessment (FMA), and TCM symptoms in acute state were completely recorded

(6) Cases with correct contacts for follow-up were included

2.5. Exclusion Criteria. Cases with any of the following exclusion criteria were excluded: (1) patients with transient ischemic attack; (2) presence of another chronic severe disease, including myocardial infarction, heart failure, renal failure, malignant tumor, severe infection, Parkinson's disease, and epilepsy; (3) patients with conscious disturbance and the Glasgow Coma Scale (GCS) score of $\leq 8$; (4) patients with severe dementia, to be specific, the MiniMental State Examination (MMSE) score of $\leq 17$ in illiterate patients, that of $\leq 20$ in patients receiving primary education, and that of $\leq 24$ in those receiving junior or higher education; (5) patients and their families who were reluctant to be followed up.

2.6. Data Collection. Medical records, including identification (such as age, sex, and contact information), past medical history, examination, stroke onset time, admission time, NIHSS scores, FMA, and TCM symptoms and signs, were collected on the date of admission. All TCM symptoms were placed in a table in Excel, and those with frequencies greater than $5 \%$ were screened for further analysis. Each screened symptom had a score from 0 to 3 according to the severity. Typically, the electronic medical record system was designed by the Shanghai Kingstar Winning Software company (EMR No. 5, the architecture of Client/Server model). Participants were invited to the hospital for follow-up by telephone at one year after stroke onset.

2.7. Intervention. In this retrospective cohort study, all patients received standard treatments [11], including basic treatments for vital signs, antiplatelet medication, intravenous thrombolytics, and mechanical thrombectomy if eligible. Additionally, acupuncture therapy and rehabilitation were also carried out at the acute phase.

Acupuncture therapies, including electroacupuncture and scalp acupuncture, were also given at the acupoints of Hegu-LI4, Shenting-DU24, Fenglong-ST40, and BaihuiDu20 as well as the motor area of the scalp for 30 min once a day (5 days/week) in the hospital. Specifically, the electroacupuncture at the paretic side was divided into two protocols, including Jianyu-LI15, Quchi-LI11, Waiguan-TE5, Yanglingquan-GB34, and Zusanli-ST36 with dilatational wave $(1.5 \sim 2.5 \mathrm{~Hz})$ protocol in the presence of flaccid paralysis; Tianjing-TE10, Naohui-TE13, Waiguan-TE5, Weizhong-BL40, and Chengshan-BL57 with dilatational wave $(2 \mathrm{~Hz})$ protocol for spastic paralysis. Sterile disposable needles $(0.25 \mathrm{~mm} \times 40 \mathrm{~mm}$ in size) were utilized in this study, which were inserted into the scalp acupoints as 
described in our prior protocol [12]. Briefly, the needles were twirled for $1 \mathrm{~min}$ (at least 200 turns per min) for once every $10 \mathrm{~min}$.

2.8. Outcome Measurement. The primary outcome was the FMA scale at one-year follow-up after stroke onset. The FMA scale is a preferred, reliable, and stable tool for the assessment of motor functional performance of patients in restorative stroke trials [13], including flexor synergy, extensor synergy, movement combining synergy, movement out of synergy, coordination, and speed. The total store of 100 reflects a normal motor function.

The secondary outcomes were the MBI scale and the mRS scale at one-year follow-up after stroke onset. The MBI is a scale that measures ten basic activities of daily life, including dressing, feeding, continence of the bladder and bowel, going to the toilet, grooming, bathing, moving, walking at $45 \mathrm{~m}$, and walking up and down stairs, which are related to self-care and mobility [14]. The normal scale is 100. In addition, the mRS scale is commonly used to measure the disability degree of patients with stroke or other neurological disability in daily activities, which has become the most extensively utilized clinical outcome measure in clinical studies [15]. The scale score ranges from 0-6 points. Favorable outcome was defined as a score from 0 to 2 on mRS at first year.

2.9. Statistical Analysis. Statistical analysis was carried out by the Statistical Product and Service Solutions (SPSS) statistical package program (version 25, SPSS Inc., Chicago, IL, USA). The quantitative data such as FMA, MBI, and NIHSS were expressed as means with standard deviation. The count data such as TCM symptoms were expressed as counts (percentage). Frequency comparisons were made with the methods of Chi-square test. Multiple regression analysis was used to determine the correlation between TCM symptoms and FMA. All variables were force-entered. Collinearity diagnostics were made to analyze multicollinearity. Durbin-Watson (DW) test was made to check the regression models. Moreover, a receiver operating characteristic (ROC) curve was drawn to verify the discriminatory power of the models.

\section{Results}

3.1. General Patient Characteristics. Eventually, 315 out of the 1650 patients at five inpatient departments of Longhua Hospital Affiliated to Shanghai university of TCM were enrolled in this retrospective cohort study in accordance with the inclusion and exclusion criteria from January 2016 to December 2017 (Figure 1).

In the overall sample, the mean age was $68.64 \pm 10.72$ years. $61.0 \%$ were males. $100(31.7 \%)$ cases had a stroke history and $104(33.0 \%)$ cases had a surgery history. In the past medical history, the first five diseases with high frequency were hypertension (76.8\%), diabetes (42.5\%), migraine $(24.8 \%)$, coronary atherosclerotic heart disease $(24.4 \%)$, and interstitial lung disease (16.5\%), respectively.
The mean scores of NIHSS, FMA, MBI, and mRS on admission were $7.51 \pm 3.52,30.65 \pm 9.60,30.66 \pm 7.76$, and $3.42 \pm 0.63$, respectively. The infarct locations mainly included basal ganglia (25.1\%), internal capsule $(18.2 \%)$, cerebellum $(11.5 \%)$, frontal lobe $(11.3 \%)$, and brain stem (10.8\%).

In medication therapy, $25(7.9 \%)$ patients received thrombolytic therapy. In antiplatelet medications, $33.8 \%$ took bayaspirin, 58.6\% took clopidogrel, and $17.9 \%$ took ticagrelor. $10.3 \%$ especially took two kinds simultaneously. After discharge, the patients continued acupuncture treatment in the outpatient department. The median course of acupuncture treatment was five (five to six) months.

In the follow-up, the mean scores of FMA and MBI at the first year were $83.25 \pm 14.73$ and $81.79 \pm 13.63$. Favorable outcome ( $n=234,74.3 \%$ ) was defined as a score from zero to two on mRS at the first year. No patients died. The overall recurrence rate was $20.3 \%$.

3.2. The Correlation between TCM Symptoms and Motor Function at One-Year after Stroke. Twenty-five TCM symptoms on admission were screened totally. Using Chisquare test, 18 TCM symptoms were related with $\mathrm{mRS}$ at the first year, which showed their sensitivity to predict motor function of $\mathrm{mRS}(P<0.05)$ (Table 1$)$.

In multivariable linear regression analysis, 10 symptoms were proved to be statistically correlated with $\mathrm{MBI}$ at the first year $(P<0.05)$. The DW statistic was 1.409 , which indicated nonautocorrelation. The R-squared value was 0.682 , which indicated the goodness of fit. The adjusted R-squared was 0.654 , which showed the regression model was reliable. Figure 2 showed the analysis of residual. Figure 3 showed a linear trend between the measured and predicted values of regression. The results of the standardized B value suggested that seven symptoms might be the primary symptoms to affect the prognosis of motor function, such as restlessness, hemiplegia, short breath, headache, and hemianesthesia (Table 2).

On the other hand, 10 symptoms were proved to be statistically correlated with FMA at the first year $(P<0.05)$ in multivariable linear regression analysis. The DW statistic was 1.446, which indicated nonautocorrelation. The $R$ squared value was 0.650 , which indicated the quality of linear fit. The adjusted $R$-squared was 0.620 , which showed the regression model was reliable. Figure 4 showed the analysis of residual. Figure 5 indicated a linear trend between the FMA and predicted values of regression. The results of the standardized B value suggested that seven symptoms might be the primary symptoms to affect the prognosis of motor function, such as restlessness, hemiplegia, short breath, headache, and constipation (Table 3).

After screening the above regression models (MBI and FMA), nine symptoms were of repeated occurrence, including hemiplegia, hemianesthesia, night sweats, short breath, headache, tinnitus, thirsty, constipation, and restlessness, which composed the third regression model. Moreover, the total scores of these three models were calculated, in contrast to the NIHSS scores at admission, 


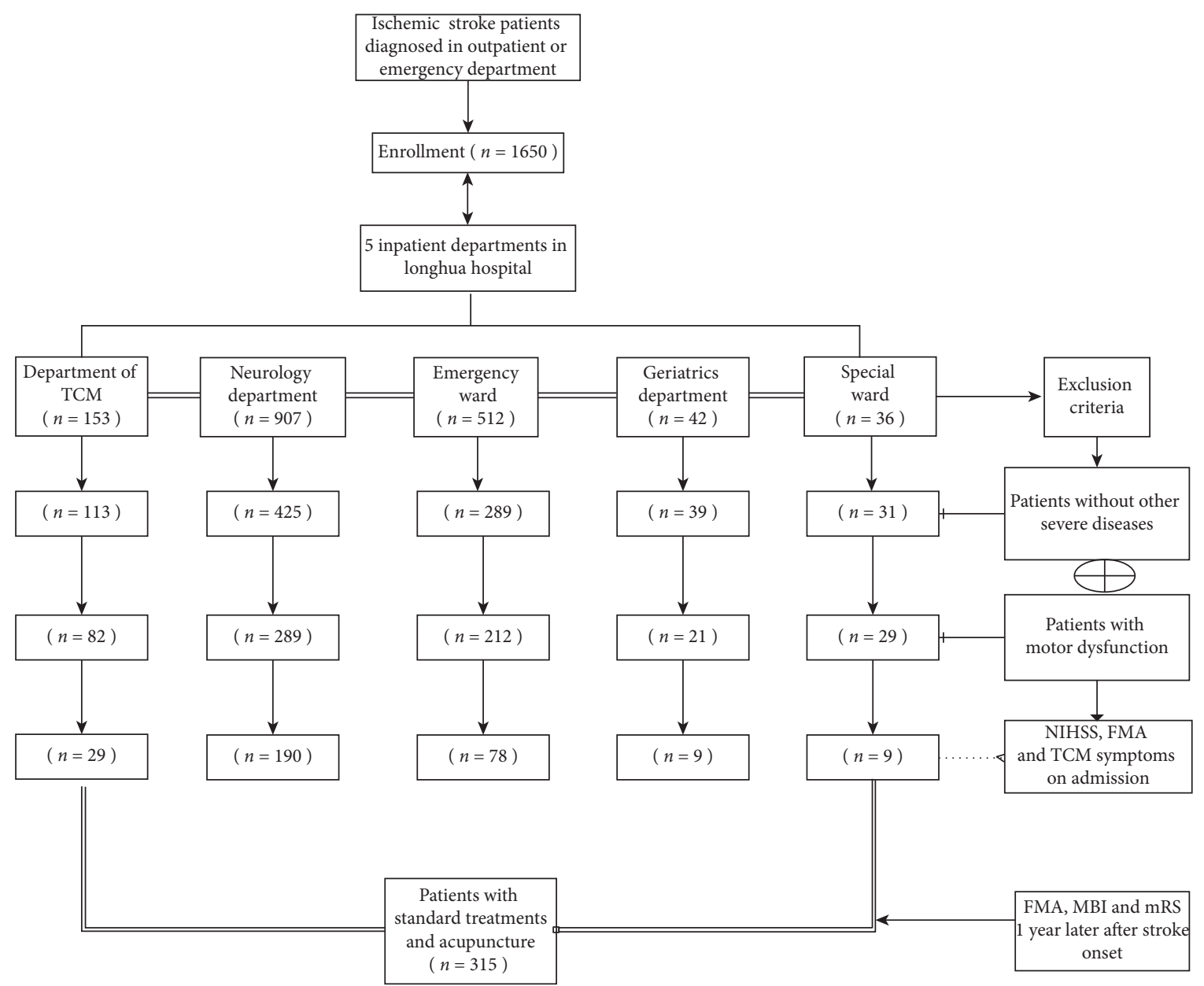

FIGURE 1: Flowchart of the study recruitment.

TABLE 1: The correlation between TCM symptoms and mRS at the first year.

\begin{tabular}{|c|c|c|c|}
\hline TCM symptoms & $\mathrm{mRS}(0 \sim 2), n(\%) n=234$ & mRS (3 5), $n(\%) n=81$ & $P$ Value \\
\hline Hemiplegia & $234(100)$ & $81(100)$ & $<0.001$ \\
\hline Nausea and vomiting & $163(69.7)$ & $71(87.7)$ & $<0.001$ \\
\hline Hemianesthesia & $43(18.4)$ & $52(64.2)$ & $<0.001$ \\
\hline Lassitude & $91(38.9)$ & $35(43.2)$ & 0.043 \\
\hline Slobbering & $46(19.7)$ & $24(29.6)$ & $<0.001$ \\
\hline Night sweat & $175(74.8)$ & $27(33.3)$ & $<0.001$ \\
\hline Short breath & $37(15.8)$ & $36(44.4)$ & $<0.001$ \\
\hline Headache & $9(3.8)$ & $34(42.0)$ & $<0.001$ \\
\hline Tinnitus & $83(35.5)$ & $3(3.7)$ & $<0.001$ \\
\hline Feverish palms and soles & $97(41.5)$ & $13(16.0)$ & $<0.001$ \\
\hline Bitter taste & $161(68.8)$ & $61(75.3)$ & $<0.001$ \\
\hline Gurgling with sputum & $29(12.4)$ & $30(37.0)$ & $<0.001$ \\
\hline Dry pharynx & $57(24.4)$ & $12(14.8)$ & 0.011 \\
\hline Thirsty & $87(37.2)$ & $11(13.6)$ & $<0.001$ \\
\hline Frequent urination & $107(45.7)$ & $29(35.8)$ & 0.007 \\
\hline Constipation & $88(37.6)$ & $35(43.2)$ & $<0.001$ \\
\hline Restlessness & $73(31.2)$ & $69(85.2)$ & $<0.001$ \\
\hline Poor appetite & $139(59.4)$ & $59(72.8)$ & 0.031 \\
\hline
\end{tabular}

Significant difference, $P<0.05$. $P$ values based on Chi-square test. 


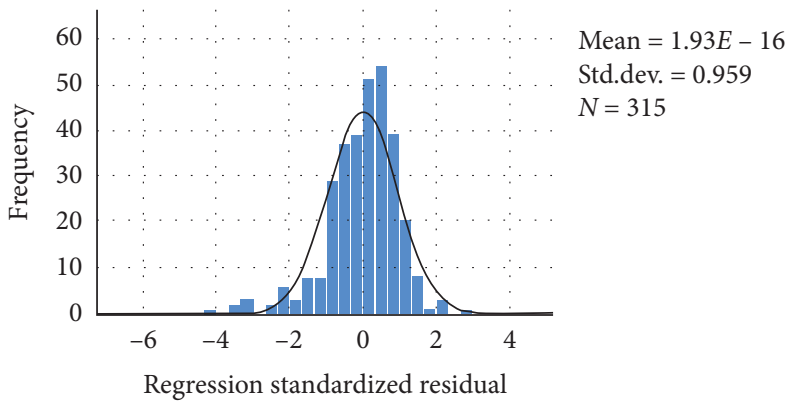

FIGURE 2: Regression standardized residual histogram (MBI).

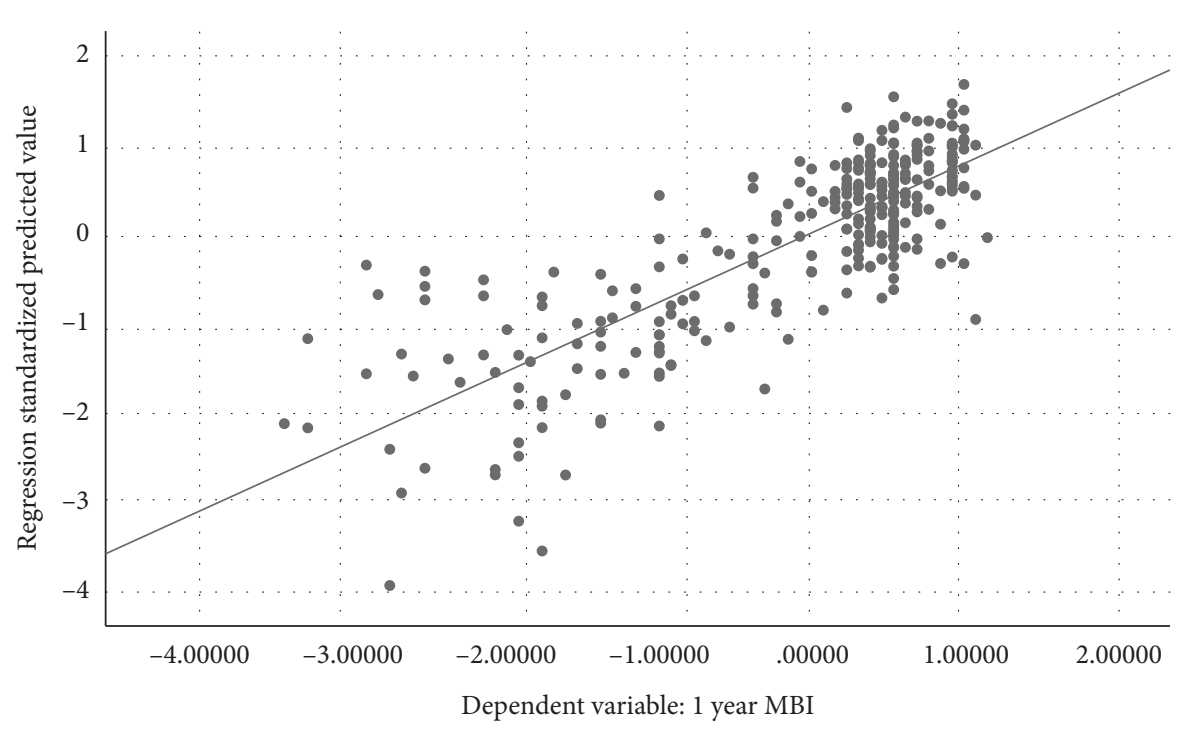

Figure 3: Regression plot (measured value vs. predicted value) $(\mathrm{MBI}) . R^{2}=0.682$.

TABLE 2: The correlation between TCM symptoms and MBI at the first year.

\begin{tabular}{lccc}
\hline TCM symptoms & $\mathrm{n}(\%)$ & St. B value $(95 \%$ CI $)$ & $P$ value \\
\hline Hemiplegia & $315(100)$ & $-0.178(-0.255,-0.102)$ & $<0.001$ \\
Restlessness & $142(45.1)$ & $-0.286(-0.371,-0.202)$ & $<0.001$ \\
Hemianesthesia & $95(30.2)$ & $-0.105(-0.181,-0.029)$ & $<.007$ \\
Short breath & $73(23.2)$ & $-0.166(-0.240,-0.093)$ & $<0.001$ \\
Headache & $43(13.7)$ & $-0.114(-0.193,-0.035)$ & 0.005 \\
Gurgling with sputum & $59(18.7)$ & $-0.071(-0.142,-0.001)$ & 0.048 \\
Constipation & $123(39.0)$ & $-0.087(-0.161,-0.012)$ & 0.023 \\
Night sweat & $202(64.1)$ & $0.109(0.032,0.186)$ & 0.006 \\
Tinnitus & $86(27.3)$ & $0.111(0.042,0.180)$ & 0.002 \\
Thirsty & $98(31.1)$ & $0.122(0.051,0.192)$ & 0.001 \\
\hline
\end{tabular}

St: standardized; CI: confidence interval; significant difference, $P<0.05$.

respectively, so as to draw the ROC curve. Figure 6 showed all the four curves were in the upper left corner closely. The results of area under the curve (AUC) showed that the sequence from high to low was MBI, FMA, repeated, and NIHSS, which indicated the higher accuracy of the three TCM scales. In sensitivity, the MBI model was the best (63\%). In specificity, the FMA model was the best. Conclusively, the MBI model with 10 symptoms had the highest value in the prognosis of motor function (Table 4).

\section{Discussion}

In this paper, multivariable linear regression analysis was used to determine the correlation between 25 obtained TCM symptoms at admission and motor function by means of MBI and FMA at the first year. Moreover, ROC curves were drawn to verify the efficiency of the regression models.

The NIHSS scale is widely used to assess neurological impairment and evaluate the prognosis of motor function in 


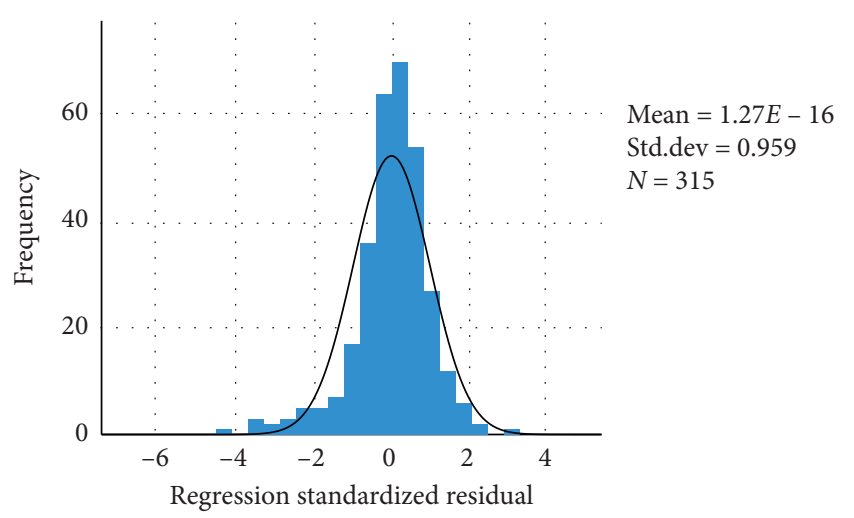

FIGURE 4: Regression standardized residual histogram (FMA).

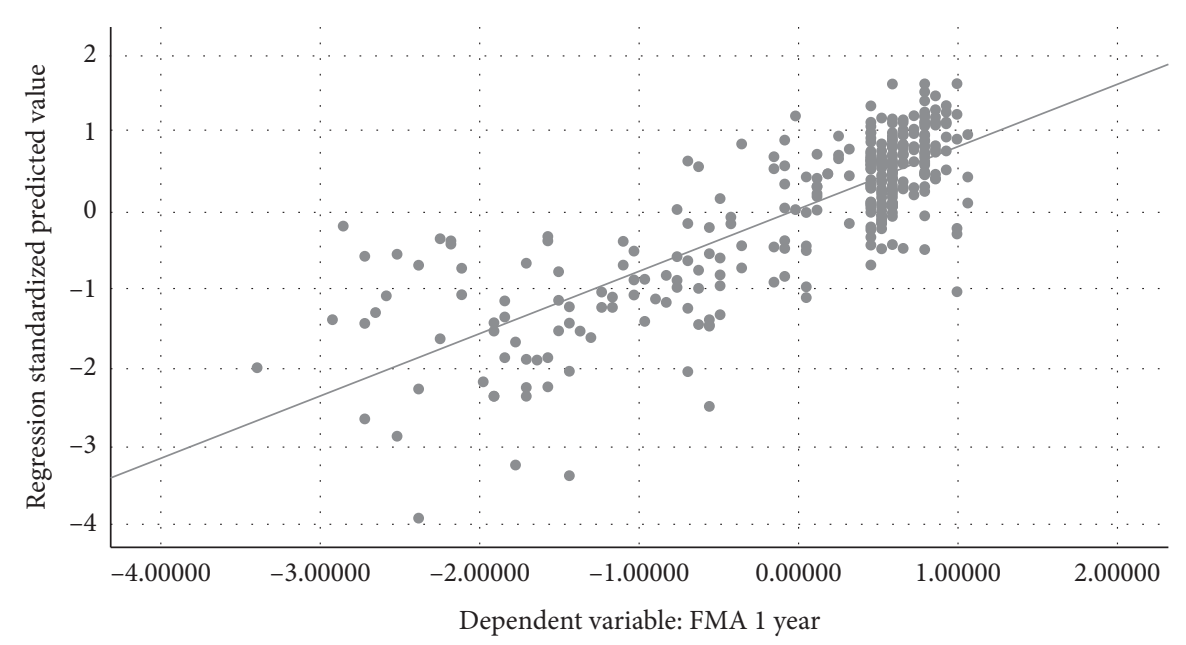

FIgURE 5: Regression plot (measured value vs. predicted value) (FMA). $R^{2}=0.650$.

TABLE 3: The correlation between TCM symptoms and FMA at the first year.

\begin{tabular}{lccc}
\hline TCM symptoms & $\mathrm{n}(\%)$ & St. B value $(95 \%$ CI $)$ & $P$ value \\
\hline Hemiplegia & $315(100)$ & $-0.176(-0.256,-0.095)$ & $<0.001$ \\
Nausea and vomiting & $234(74.3)$ & $-0.080(-0.155,-0.005)$ & 0.037 \\
Hemianesthesia & $95(30.2)$ & $-0.100(-0.180,-0.021)$ & 0.014 \\
Short breath & $73(23.2)$ & $-0.134(-0.212,-0.057)$ & 0.001 \\
Headache & $43(13.7)$ & $-0.115(-0.198,-0.031)$ & 0.007 \\
Constipation & $123(39.0)$ & $-0.097(-0.175,-0.019)$ & 0.015 \\
Restlessness & $142(45.1)$ & $-0.287(-0.376,-0.198)$ & $<0.001$ \\
Tinnitus & $86(27.3)$ & $0.091(0.018,0.163)$ & 0.015 \\
Thirsty & $98(31.1)$ & $0.099(0.025,0.173)$ & 0.009 \\
Night sweat & $202(64.1)$ & $0.122(0.041,0.204)$ & 0.003 \\
\hline
\end{tabular}

St: standardized; CI: confidence interval; significant difference, $P<0.05$.

acute stroke as well. The NIHSS scale consists of 11 items, including consciousness, eye movement, visual field, facial symmetry, body movement, mutual movement, sensation, and language function, with good validity and reliability [16]. However, some important factors closely related to prognosis are neglected, which might reduce the efficiency in evaluation [17]. The results indicated three TCM models with reasonable accuracy to have ROC curves in the upper left triangle above the reference line (Figure 6). Generally, the AUC can be thought of an indicator of overall "accuracy." Considering an AUC of 0.767 , the model of MBI with 10 symptoms had the highest value and best discrimination, although the specificity of the model of FMA is higher. Since the primary intended use of the prognostic test is either "ruling-in" or "ruling-out" a target condition, the balance between false-positive and false-negative rates is clinically important. In order to find the optimal trade-off between sensitivity and specificity, a value of $\geq 8$ represents this point 


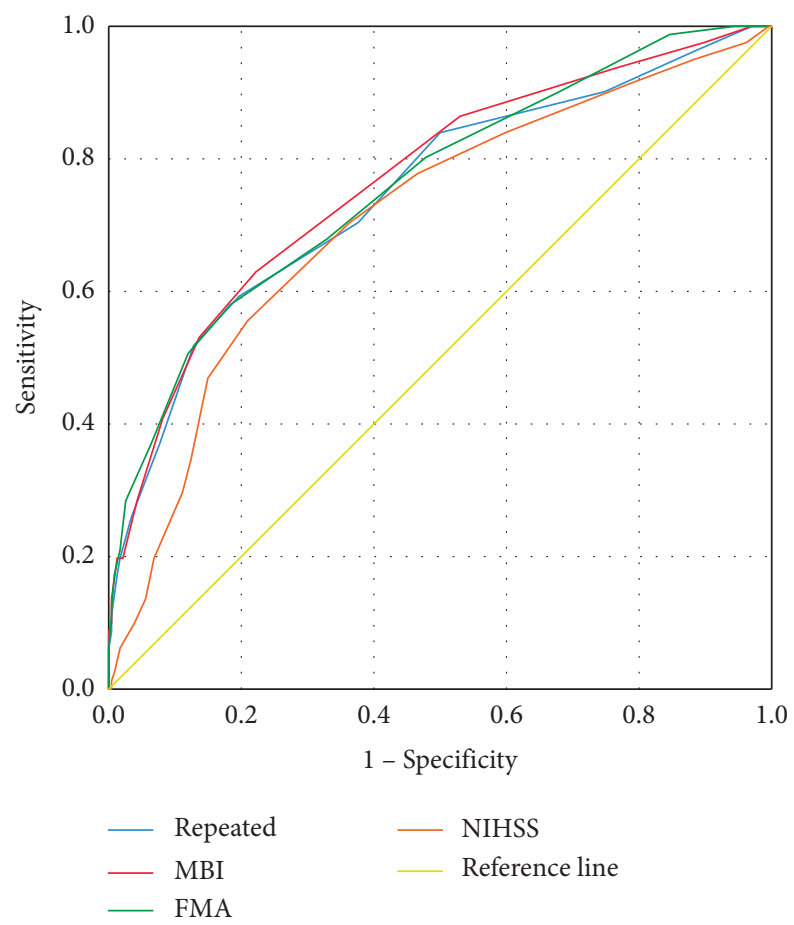

FIGURE 6: ROC curve on four scales.

TABle 4: The results of ROC curve.

\begin{tabular}{|c|c|c|c|c|c|c|}
\hline Model & AUC $(95 \% \mathrm{CI})$ & Youden index & Critical value & Sensitivity (\%) & Specificity (\%) & $P$ value \\
\hline NIHSS & $0.711(0.644,0.778)$ & 0.347 & 9 & 55.6 & 79.1 & $<0.001$ \\
\hline MBI & $0.767(0.705,0.829)$ & 0.408 & 8 & 63 & 77.8 & $<0.001$ \\
\hline FMA & $0.761(0.698,0.823)$ & 0.396 & 9 & 58 & 81.6 & $<0.001$ \\
\hline Repeated & $0.749(0.684,0.815)$ & 0.396 & 8 & 59.3 & 80.3 & $<0.001$ \\
\hline
\end{tabular}

AUC: area under the curve; CI: confidence interval; significant difference, $P<0.05$.

of balance, which indicates that the sensitivity in predicting motor dysfunction at the first year after stroke is $63 \%$ and the specificity is $77.8 \%$. Meanwhile, while the critical value of NIHSS is nine, the sensitivity in predicting bad outcome is $55.6 \%$ and the specificity is $79.1 \%$. Therefore, the TCM scale of MBI model is better in balance. In these 10 symptoms, hemiplegia is the primary symptom of stroke; hemianesthesia and headache are the specific symptoms of stroke; and the other seven symptoms, such as short breath, gurgling with sputum, constipation, and restlessness, are accompanied symptoms. For example, patients with restlessness (St. $B=-0.286,95 \% \mathrm{CI}-0.371$ to -0.202 ) on the first day were inclined to get poor recovery.

Generally, the ability to predict outcome in patients with acute stroke is of research and clinical value [18]. Previous stroke, age, and urinary or bowel incontinence on admission are adverse prognostic indicators of function [19]. Particularly, the severity of paresis, presence of hemianopia, reduced leg power, and size of brain lesion are all predictive of walking within 30 days after stroke [18]. Moreover, urinary incontinence, severity of hemiplegia, comorbidity, consciousness at admission, cognitive status, and depression are independent factors that are associated with the outcome in terms of activities of daily living (ADL) beyond six months [20]. The establishment of an adequate prognosis will increase the efficiency of stroke services and reduce costs. TCM symptom, as a kind of PROs, has been widely used in clinical practice for many years. The initial severity of specific symptoms and accompanied symptoms observed in the first day after stroke are important indicators of outcome at one year after stroke in this study.

Our previous studies $[21,22]$ found that wind syndrome and phlegm syndrome are the major single syndromes occurring within 30 days of onset. This study made further investigation into the prognostic value of definite symptoms occurring on the first day. For instance, gurgling with sputum, which is a reflection of phlegm syndrome, might be a factor affecting the recovery of motor dysfunction. A previous study [7] used the Pearson correlation test and logistic regression model to review 489 patients with acute ischemic stroke and finally screened eight items of the scale, indicating that early appearance of anxiety might predict a bad outcome at the ninetieth $\left(90^{\text {th }}\right)$ day (OR: 3.17). Our study observed the recovery of motor function at the first year, which was much longer. The symptoms collected were 
based on the theory of TCM differentiation. For the concept of holism, we took all the patient-reported symptoms as a whole, by the use of multivariable linear regression analysis accompanied with the ROC curve. Therefore, the results of the predictive model were reliable, which could provide a basis for the development of TCM scales.

Some limitations should be noted in this study. Considering that the study was retrospective, the quantity of the collected information depended on the quality of medical records. Inadequate information made it difficult to assess principal component analysis for classification. However, these TCM symptoms at the early stage of stroke might be valuable for the establishment of prognostic scales. Besides, we could not observe the improvements of TCM symptoms dynamically after treatment due to the limitation of the literature. The regression model here just reflected the first day of admission. We will focus on this part in further research.

\section{Conclusion}

It is observed in this study that, through the one-year followup, once the ischemic stroke onset occurred, the combination of 10 TCM symptoms, including hemiplegia, restlessness, hemianesthesia, short breath, headache, constipation, night sweat, tinnitus, thirsty and gurgling with sputum, may affect the recovery of motor dysfunction. Of them, seven symptoms, including restlessness, hemiplegia, short breath, headache, hemianesthesia, constipation, and gurgling with sputum, might be the primary symptoms.

\section{Abbreviations}

ADL: Activities of daily living

AUC: $\quad$ Area under the curve

DALYs: Disability-adjusted life-years

DW: Durbin-watson

FMA: Fugl-Meyer assessment

GCS: $\quad$ Glasgow coma scale

hr-QOL: Health-related quality of life

MBI: $\quad$ Modified Barthel index

MMSE: Mini-mental state examination

mRS: $\quad$ Modified Rankin scale

NIHSS: National Institutes of Health Stroke Scale

PROs: Patient-reported outcomes

ROC: $\quad$ Receiver operating characteristic

SPSS: $\quad$ Statistical product and service solutions

SSTCM: Stroke scales of traditional Chinese medicine

TCM: Traditional Chinese medicine.

\section{Data Availability}

All data included in this study are available upon request through contacting the corresponding author.

\section{Ethical Approval}

The study was approved by the Ethics Committee of Longhua Hospital Affiliated to Shanghai University of TCM (2014KL-006).

\section{Conflicts of Interest}

The authors declare that they have no competing interests.

\section{Authors' Contributions}

All authors have participated extensively in the study and had proof-read the final manuscript. Preparation and review were done by $\mathrm{Xu} \mathrm{J}$, Pei J, Fu QH, and Zhan YJ. Pei J was responsible of supervision and coordination of clinical trials. $\mathrm{Xu} \mathrm{J}$ and Zhan YJ were responsible for subject recruitment. $\mathrm{Xu} \mathrm{J}$ and $\mathrm{Fu} \mathrm{QH}$ were responsible or subject assessment. Xu J, Zhan YJ, and $\mathrm{Fu} \mathrm{QH}$ were responsible for subject treatment. $\mathrm{Xu} \mathrm{J}$ and $\mathrm{Fu} \mathrm{QH}$ were responsible for statistical analysis. All authors read and approved the final manuscript.

\section{Acknowledgments}

Thanks are due to all the participants and clinical researchers involved in the publications cited in this study. This article was supported by the Financing Scheme of Arising Interdisciplinary Subjects of TCM in Shanghai (Shxxjcxk201709), the TCM Genre Program of Shanghai Health Bureau (ZY (2018-2020)-CCCX-1006), the Key Scientific Research Program of Shanghai Municipal Science and Technology Committee in China (16401970300 and 18401970500), and the National Natural Science Foundation of China (grant number: 81603697).

\section{References}

[1] S. Wu, B. Wu, M. Liu et al., "Stroke in China: advances and challenges in epidemiology, prevention, and management," The Lancet Neurology, vol. 18, no. 4, pp. 394-405, 2019.

[2] V. L. Feigin, G. Nguyen, K. Cercy et al., "Global, regional, and country-specific lifetime risks of stroke, 1990 and 2016," New England Journal of Medicine, vol. 379, no. 25, pp. 2429-2437, 2018.

[3] V. L. Feigin, B. Norrving, and G. A. Mensah, "Global burden of stroke," Circulation Research, vol. 120, no. 3, pp. 439-448, 2017.

[4] L. D. Wang, J. M. Liu, Y. Yang, and Y. L. Wang, "Chinese prevention and treatment of stroke 2017," Chinese Journal of Cerebrovascular Diseases, vol. 15, no. 11, pp. 611-617, 2018.

[5] C. H. Yu, Y. N. Sun, L. Y. He, W. J. Bai, and B. Y. Liu, "Traditional Chinese medicine generic questionnaire for life quality: a systematic review," Chinese Journal of Traditional Chinese Medicine and Pharmacy, vol. 31, no. 02, pp. 432-437, 2016.

[6] D. R. Wu, S. L. Lai, X. F. Guo, Z. H. Wen, and W. X. Liang, "Reliability and validity of Health Scale of Traditional Chinese Medicine," Journal of Chinese Integrative Medicine, vol. 6, no. 7, pp. 682-689, 2008.

[7] K. G. Cao, C. H. Fu, H. Q. Li, X. Y. Xin, and Y. Gao, "A new prognostic scale for the early prediction of ischemic stroke recovery mainly based on traditional Chinese medicine symptoms and NIHSS score: a retrospective cohort study," BMC Complementary and Alternative Medicine, vol. 15, p. $407,2015$.

[8] R. E. Sherman, S. A. Anderson, G. J. Dal Pan et al., "Realworld evidence-what is it and what can it tell us?" New 
England Journal of Medicine, vol. 375, no. 23, pp. 2293-2297, 2016.

[9] Chinese Society of Neurology and Chinese Stroke Society, "Chinese guidelines for diagnosis and treatment of acute ischemic stroke 2014," Chinese Journal of Neurology, vol. 4, no. 48, 2015.

[10] Z. Yi, Y. Da, and X. Bao, "The Encephalopaty Accident and Emergency Collaborative Collaborativ Group of State Administration of TCM," Criteria of diagnosis and therapeutic effect evaluation for apoplexy (trial version). Beijing, vol. 19, pp. 55-56, 1996.

[11] W. J. Powers, A. A. Rabinstein, T. Ackerson et al., "Guidelines for the early management of patients with acute ischemic stroke: a guideline for healthcare professionals from the American heart association/American stroke association," Stroke, vol. 49, no. 3, pp. e46-e110, 2018.

[12] J. Wang, J. Pei, D. Khiati et al., "Acupuncture treatment on the motor area of the scalp for motor dysfunction in patients with ischemic stroke: study protocol for a randomized controlled trial," Trials, vol. 18, no. 1, p. 287, 2017.

[13] J. See, L. Dodakian, C. Chou et al., "A standardized approach to the fugl-meyer assessment and its implications for clinical trials," Neurorehabilitation and Neural Repair, vol. 27, no. 8, pp. 732-741, 2013.

[14] S. O. C. Leung, C. C. H. Chan, and S. Shah, "Development of a Chinese version of the modified Barthel index - validity and reliability," Clinical Rehabilitation, vol. 21, no. 10, pp. 912-922, 2007.

[15] J. L. Saver, B. Filip, S. Hamilton et al., "Improving the reliability of stroke disability grading in clinical trials and clinical practice," Stroke, vol. 41, no. 5, pp. 992-995, 2010.

[16] K. Prasad, D. Dash, and A. Kumar, "Validation of the Hindi version of national institute of health stroke scale," Neurology India, vol. 60, no. 1, pp. 40-44, 2012.

[17] T. Brott, H. P. Adams Jr., C. P. Olinger et al., "Measurements of acute cerebral infarction: a clinical examination scale," Stroke, vol. 20, no. 7, pp. 864-870, 1989.

[18] L. E. Craig, O. Wu, J. Bernhardt, and P. Langhorne, "Predictors of poststroke mobility: systematic review," International Journal of Stroke, vol. 6, no. 4, pp. 321-327, 2011.

[19] L. Jongbloed, "Prediction of function after stroke: a critical review," Stroke, vol. 17, no. 4, pp. 765-776, 1986.

[20] G. Kwakkel and B. J. Kollen, "Predicting activities after stroke: what is clinically relevant?" International Journal of Stroke, vol. 8, no. 1, pp. 25-32, 2012.

[21] Y. Song, J. Pei, Z. D. Liu et al., "Dynamic changes in traditional Chinese medicine syndromes in patients with ischemic stroke treated by acupuncture," Journal of Chinese Integrative Medicine, vol. 7, no. 4, pp. 334-341, 2009.

[22] J. Pei, L. J. Sun, R. X. Chen, T. M. Zhu, Y. Z. Qian, and D. J. Yuan, "The effect of electro-acupuncture on motor function recovery in patients with acute cerebral infarction: a randomly controlled trial," Journal of Traditional Chinese Medicine, vol. 21, no. 4, pp. 270-272, 2001. 